\title{
THE BRAUER GROUP OF AN AMITSUR FIELD
}

\author{
CHAN-NAN CHANG
}

ABstract. In this paper we compute the entire Brauer groups of Amitsur fields (i.e. certain fields of formal power series $K_{m}$ described in \$1); these are interesting generalizations of the Brauer groups over the fields of local class field theory.

1. Power series fields. Throughout this paper, we assume $K$ is a field of characteristic zero. Denote by $K\{t\}$ the field of all formal power series $p\{t\}=\sum_{v \geqq r} a_{v} t^{v}, a_{v} \in K, r>-\infty$. Let $t_{1}, \cdots, t_{m}$ be a finite set of indeterminates over $K$. Define successively, $K_{0}=K, K_{1}=K_{0}\left\{t_{1}\right\}, K_{j}=K_{j-1}\left\{t_{j}\right\}=$ $K\left\{t_{1}\right\}\left\{t_{2}\right\} \cdots\left\{t_{j}\right\}$. Thus the general element of $K_{j}$ has the form:

$$
p\{t\}=p\left\{t_{1}, \cdots, t_{j}\right\}=\sum_{v_{j}>r_{j} j} \sum_{v_{j-1} \geqq r_{j-1}} \cdots \sum_{v_{1} \geqq r_{1}} \alpha_{v_{1} v_{2} \cdots v_{j}} t_{1}^{v_{1}} t_{2}^{v_{2}} \cdots t_{j}^{v_{j}}
$$

where $r_{i}=r_{i}\left(v_{i+1}, \cdots, v_{j}\right)>-\infty$ for $i=1, \cdots, j-1, r_{j}>-\infty$. Clearly, each $K_{j}$ is a field.

If $K$ is an algebraically closed field, the power series field $K_{m}$ is called an Amitsur field of $m$-indeterminates (or simply an Amitsur field). Throughout this paper we assume $K$ is an algebraically closed field and $K_{m}$ is an Amitsur field. Let

$$
p\{t\}=\sum_{v_{m} \geqq r_{m}} \sum_{v_{m-1} \geqq r_{m-1}} \cdots \sum_{v_{1} \geqq r_{1}} \alpha_{v_{1} v_{2} \cdots v_{m}} t_{1}^{v_{1}} t_{2}^{\nu_{2}} \cdots t_{m}^{v_{m}} \in K_{m} .
$$

The smallest exponent $v_{m}$ of $t_{m}$ occurring in any nonzero terms of (1) is called the order of $p\{t\}$ and denoted by $o_{m}(p\{t\})$. Define a valuation ||$_{m}$ on $K_{m}$ as follows: $|p\{t\}|_{m}=e^{-o_{m}(p\{t\})}$ where $e$ is any real number greater than 1. Then ||$_{m}$ is a discrete, nonarchimedian valuation with the ring of integers $\mathfrak{D}_{m}=K_{m-1}\left[\left[t_{m}\right]\right]$ (the ring of formal powers series over $K_{m-1}$ ); maximal ideal $\mathfrak{P}_{m}=\left\{p\{t\} \in \mathfrak{D}_{m} \mid o_{m}(p\{t\}) \geqq 1\right\}$ and the residue class field $R_{m}=D_{m} / \mathfrak{P}_{m} \cong K_{m-1}$. It is easy to see that $K_{0}$ has trivial valuation $\mid l_{0}$; $K_{1}$ is a quasi-finite field (i.e. a field which has no inseparable extension and, for each $n$, it has exactly one extension of degree $n$ in its algebraic closure)

Received by the editors September 14, 1972.

AMS (MOS) subject classifications (1970). Primary 10C05, 10E25; Secondary 10C30, $20 \mathrm{G} 25$.

Key words and phrases. Amitsur field, Brauer group, character group, crossed product, cyclic algebra, division algebra, regular local field. 
and $K_{2}$ is a regular local field (i.e. a field which is complete under a discrete, nonarchimedean valuation with a residue class field which is quasifinite).

2. The character group of $K_{m}$. Denote the algebraic closure of the field $L$ by $L^{c}$ and the Galois group of $L^{c}$ over $L$ by $G(L)$.

Let $Z$ be the Artin group which is the completion of $Z$ with the topology of subgroups of finite index. Then $\hat{Z}$ is a compact, totally disconnected group which is identical with the projective limit of $Z / n Z$ and is canonically isomorphic to the product $\Pi Z_{p}$ over all primes $p, Z_{p}$ the ring of $p$ adic integers. If $L$ is a quasi-finite field, then it follows easily that $G(L)=\hat{Z}$ [5, §2, p. 198].

By a character over the field $L$, we mean a homomorphism of $G(L)$ into $Q / Z$ (=the rationals mod 1 ) which is continuous (Krull topology). Let $\chi(L)$ be the group of these characters over $L$. If $L$ is a quasi-finite field, then it follows from [5, Proposition 1 and Corollary, p. 197] that $\chi(L) \cong$ $Q / Z$. In the following, we use induction on $m$ and the above result to find $\chi\left(K_{m}\right)$.

2.1 Lemma. Let $p\{t\}=1+\sum_{i=1} \alpha_{i} t_{m}^{i} \in K_{m}$ where $\alpha_{i} \in K_{m-1}$. Then $p\{t\} \in$ $K_{m}^{n}=\left\{\alpha^{n} \mid \alpha \in K_{m}\right\}$ for every $n \in Z^{+}$.

PROOF. Let $q\{t\}=1+\sum_{i=1}^{\infty} \beta_{i} t_{m}^{i} \in K_{m}$ where $\beta_{i} \in K_{m-1}$; then $q\{t\}^{n}=$ $1+\sum_{i=1}^{\infty} r_{i} t_{m}^{i}$ where $r_{i} \in K_{m-1}$ and $r_{i}=r_{i}^{\prime}\left(\beta_{1}, \beta_{2}, \cdots, \beta_{i-1}\right)+n \beta_{i}$ is a function of $\beta_{1}, \beta_{2}, \cdots, \beta_{i}$. Hence there exist $\beta_{i}, i=1,2, \cdots$, such that $r_{i}=\alpha_{i}$ for all $i$. Therefore, $p\{t\}=q\{t\}^{n} \in K_{m}^{n}$.

2.2 Proposition. Every finite extension of $K_{m}$ is of the form $K_{m}(A, T)$ where $A$ is algebraic over $K_{m-1}$ and $T^{n}=\alpha t_{m}$ for some $n \in Z^{+}$and $\alpha \in K_{m-1}$.

PRoof. $K_{m}=K_{m-1}\left\{t_{m}\right\}$ is complete with respect to the valuation ||$_{m}$. The subfield $K_{m-1}$ is a set of representatives for the residue class field and since the characteristic is 0 , it follows easily from Hensel's lemma that if $L / K_{m}$ is finite algebraic, then its maximal unramified subfield is of the form $K_{m}(A)$ with $A$ algebraic over $K_{m-1}$. Then $L / K_{m}(A)$ is pure ramified, hence generated by an element $\theta$ with $\left|\theta^{n}\right|_{m}=\left|t_{m}\right|_{m}$ where $n$ is the ramification number; i.e. $\theta^{n}=\alpha(1+\pi) t_{m}$ with $\alpha \in K_{m-1}$ and $\pi$ an element of $L$ with $|\pi|<1$ in the unique extension of ||$_{m}$ to $L$.

By Lemma 2.1, which applies here because $L$ is a field of formal power series in $\theta$, there is a $\gamma \in L$ with $\gamma^{n}=1+\pi$. Set $T=\theta \gamma^{-1}$; then $L=$ $K_{m}(A, T)$. Q.E.D.

By induction on $m$ and Proposition 2.2 we get:

2.3 Proposition. $K_{m}^{c}$ is generated by adjoining roots of the equations $\chi^{n}=t_{i}$, where $n$ is any positive integer and $i=1, \cdots, m$. 
2.4 Proposition. $G\left(K_{m}^{c} / K_{m}\right) \cong \bigoplus_{i=1}^{m} \hat{Z}$.

Proof. Let $\sigma$ be any automorphism of $K_{m}^{c} / K_{m}$; it induces an automorphism $\sigma_{0}$ on $K_{m-1}^{c} / K_{m-1}$ and it is easy to see that we can extend $\sigma_{0}$ to an automorphism $\sigma^{\prime}$ of $K_{m}^{c} / K_{m}$ by defining $\sigma^{\prime}\left(T_{n}\right)=T_{n}$ for every $T_{n}$ such that $T_{n}^{n}=t_{m}$; i.e. $\sigma^{\prime}$ has the same effect as $\sigma$ on $K_{m-1}^{c}$ but acts as identity on all the elements $T^{n}$. One easily sees that $\sigma=\sigma^{\prime \prime} \sigma^{\prime}$ where $\sigma^{\prime \prime}$ is the identity on $K_{m-1}^{c}\left\{t_{m}\right\}$ and maps each $T_{n}$ into $\zeta(\sigma, n) T_{n}$ where $\zeta(\sigma, n)$ is an $n$th root of unity. Since all roots of unity are in $K_{0}$, it follows that $\sigma^{\prime \prime}$ and $\sigma^{\prime}$ commute. Then automorphisms $\sigma^{\prime}$ form a subgroup isomorphic to $\hat{Z}$ and $\sigma^{\prime \prime}$ a subgroup isomorphic to $G\left(K_{m-1}^{c} / K_{m-1}\right)$. So our proposition follows.

3. The Brauer group of the Amitsur field $K_{m}$. Denote the Brauer group of the field $L$ by $B(L)$. The following theorem of Witt [6] is well known: Let $L$ be a local field (i.e. a field which is complete in the topology induced by a discrete, nonarchimedean valuation) with residue class field $L$ which is perfect. Then the following sequence, $\{1\} \rightarrow B(L) \rightarrow B(L) \rightarrow \chi(G(L)) \rightarrow\{1\}$, is exact and split. In what follows we use induction on $m$ and the theorem of Witt to determine the structure of $B\left(K_{m}\right)$.

3.1 THEOREM. $B\left(K_{m}\right) \cong \bigoplus_{i=1}^{(m-1) m / 2} Q / Z$ for every nonnegative integer $m$.

Proof. Since $K_{0}$ is algebraically closed and $K_{1}$ is a $c_{1}$-field, hence $B\left(k_{0}\right)=B\left(K_{1}\right)=\{1\}$ (see [5, p. 170]). Suppose that $m>1$. Since $R_{m}=K_{m-1}$ with respect to the valuation ||$_{m}$, it follows from the theorem on Witt that

$$
B\left(K_{m}\right) \cong B\left(K_{m-1}\right) \oplus \chi\left(G_{c}\left(K_{m-1}\right)\right) \text {. }
$$

By Proposition 2.3, $\chi\left(G_{c}\left(K_{m-1}\right)\right) \cong \bigoplus_{i=1}^{m-1} Q / Z$. By the induction hypothesis, $B\left(K_{m-1}\right) \cong \bigoplus_{i=1}^{(m-2)(m-1) / 2} Q / Z$. Therefore

$$
B\left(K_{m}\right) \cong \bigoplus_{i=1}^{(m-2)(m-1) / 2} Q / Z \oplus \bigoplus_{i=1}^{m-1} Q / Z \cong \bigoplus_{i=1}^{(m-1) m / 2} Q / Z \text {. Q.E.D. }
$$

4. The generators of $B\left(K_{m}\right)$. Let $L$ be a field which contains an $n$th primitive root of unity $\zeta_{n}$ and $\alpha, \beta \in L$. Let $(\alpha, \beta)_{n}$ be the cyclic $L$-algebra of degree $n$ which is generated by $\chi, y$ with $\chi^{n}=\alpha, y^{n}=\beta$ and $\chi y=\zeta_{n} y \chi$.

Let $u, v$ be two central simple algebras over $L$. If $u, v$ represent the same element in $B(L)$, then we write $u \sim v$.

4.1 Lemma. Let $(\alpha, \beta)_{n},(\alpha, \gamma)_{n}$ be two cyclic L-algebras. Then

$$
(\alpha, \beta)_{n} \times(\alpha, \gamma)_{n} \sim(\alpha, \beta \gamma)_{n} .
$$

Proof. See [1, Theorem 11, p. 74].

4.2 Proposition. Let $L$ be a cyclic extension of degree $n$ over $K_{m}$. Then there exists $t \in L$ such that $t^{n}=t_{1}^{\gamma_{1}} t_{2}^{\gamma_{2}} \cdots t_{m}^{\gamma_{m}}$ where $\gamma_{i} \in Z$ and $L=K_{m}(t)$. 
Proof. Since $K_{m}$ contains $n$th roots of unity, it follows from Hilbert's Theorem 90 that there exists $u \in L$ such that $u^{n} \in K_{m}$ and $L=K_{m}(u)$. Let $u^{n}=\sum_{i=\gamma_{m}}^{\infty} u_{i} t_{m}^{i}$ where $u_{i} \in K_{m-1}, u_{\gamma_{m}} \neq 0$.

$$
u^{n}=u_{\gamma_{m}} t_{m}^{\gamma_{m}}\left(1+\sum_{i=1}^{\infty} u_{\gamma_{m}}^{-1} u_{\gamma_{m+1}} t_{m}^{i}\right)
$$

By Lemma 2.1, there exists $q\{t\} \in K_{m}$ such that $q\{t\}^{n}=1+\sum_{i=1}^{\infty} u_{\gamma_{m+i}} t_{m}^{i}$. Choose $v=q\{t\}^{-1} u$, then $v^{n}=u_{\gamma_{m}} t_{m}^{\gamma_{m}}$ and $L=K_{m}(v)$. Now $u_{\gamma} \in K_{m-1}$. By using the same method successively, it follows that there exist $t \in L$ such that $L=K_{m}(t)$ and $t^{n}=t_{1}^{\gamma_{1}} t_{2}^{\gamma_{2}} \cdots t_{m}^{\gamma_{m}}$.

4.3 THEOREM. $\left\{\left(t_{i}^{\gamma}, t_{j}\right)_{n} \mid 1 \leqq i<j \leqq m, 1 \leqq \gamma<n, n \in Z^{+}\right\}$is a set of generators of $B\left(K_{m}\right)$.

Proof. Use induction on $m$. If $m=0,1$, then $B\left(K_{0}\right)=B\left(K_{1}\right)=\{1\}$, the theorem is proved. Suppose $m>1$ and $A$ is a central simple algebra over $K_{m}$. It follows from a theorem of Witt [6] that there exists a central simple algebra $B$ over $K_{m-1}$ and a cyclic algebra $C$ over $K_{m}$ such that $A \sim B \cdot C$ where $C$ is generated by $\tau, \sigma$ with $\tau^{n}=t_{m}, K_{m-1}(\sigma)$ is a cyclic extension of degree $n$ over $K_{m-1}$ and $\tau \sigma=\zeta_{n} \sigma \tau$ where $\zeta_{n}$ is an $n$th root of unity. By Proposition 4.3, $\sigma$ can be chosen such that $\sigma^{n}=t_{1}^{\gamma_{1}} t_{2}^{\gamma_{2}} \cdots t_{m-1}^{\gamma_{m-1}}, \gamma_{i} \in Z$. Thus,

$$
C=\left(t_{1}^{\gamma_{1}} t_{2}^{\gamma_{2}} \cdots t_{m-1}^{\gamma_{m-1}}, t_{m}\right)_{n} .
$$

By Lemma 4.1, $C \sim X_{i-1}^{m-1}\left(t_{i}^{\gamma_{i}}, t_{m}\right)_{n}$. On the other hand, it follows from the induction hypothesis that $B$ can be written as a product of $\left(t_{i}^{\gamma}, t_{j}\right)_{n}$ with $1 \leqq i<j \leqq m-1,1 \leqq \gamma<n$ and $n \in Z^{+}$. Hence the theorem is proved. Q.E.D.

ACKNOWLedgement. I would like to thank Professor George Whaples for many helpful suggestions concerning the preparation of this paper.

\section{REFERENCES}

1. A. A. Albert, Structure of algebras, Amer. Math. Soc. Colloq. Publ., vol. 24, Amer. Math. Soc., Providence, R.I., 1936.

2. S. A. Amitsur, On central division algebras (to appear).

3. E. Artin, Algebraic numbers and algebraic functions, Gordon and Breach, New York, 1967. MR 38 \#5742.

4. D. G. Northcott, An introduction to homological algebra, Cambridge Univ. Press, New York, 1960. MR 22 \#9523.

5. J.-P. Serre, Corps locaux, Actualités Sci. Indust., no. 1296, Hermann, Paris, 1962. MR 27 \#133.

6. E. Witt, Schiefkörper über diskret bewerteten Körpern, J. Reine Angew. Math. 176 (1936), 153-156.

Department of Mathematics, University of Massachusetts, Amherst, MasSACHUSETTS 01002 